\title{
Threshold pion electroproduction at large momentum transfers
}

\section{V.M. Braun ${ }^{* a}{ }^{a}$ D.Yu. Ivanov, ${ }^{a b}$ A. Lenz ${ }^{a}$ and A. Peters ${ }^{a}$}

${ }^{a}$ Institut für Theoretische Physik, Universität Regensburg, D-93040 Regensburg, Germany

${ }^{b}$ Sobolev Institute of Mathematics, 630090 Novosibirsk, Russia

E-mail: vladimir.braun@physik.uni-regensburg.de, d-ivanov@math.nsc.ru,

alexander.lenz@physik.uni-regensburg.de

We consider pion electroproduction on a proton target close to threshold for $Q^{2}$ in the region $1-10 \mathrm{GeV}^{2}$. The momentum transfer dependence of the S-wave multipoles at threshold, $E_{0+}$ and $L_{0+}$, is calculated using light-cone sum rules. Predictions for the expected cross sections in the near-threshold region are presented based on a simple model taking into account $S$ - and $P$-waves.

6th International Workshop on Chiral Dynamics

July 6-10 2009

Bern, Switzerland

\footnotetext{
${ }^{*}$ Speaker.
} 


\section{Threshold Pion Production}

Pion electroproduction at threshold from a proton target

$$
\begin{aligned}
& e(l)+p(P) \rightarrow e\left(l^{\prime}\right)+\pi^{+}(k)+n\left(P^{\prime}\right), \\
& e(l)+p(P) \rightarrow e\left(l^{\prime}\right)+\pi^{0}(k)+p\left(P^{\prime}\right)
\end{aligned}
$$

can be described in terms of two generalised form factors defined as [1, 2]

$$
\begin{aligned}
& \left\langle N\left(P^{\prime}\right) \pi(k)\left|j_{\mu}^{e m}(0)\right| p(P)\right\rangle= \\
& =-\frac{i}{f_{\pi}} \bar{N}\left(P^{\prime}\right) \gamma_{5}\left\{\left(\gamma_{\mu} q^{2}-q_{\mu} q\right) \frac{1}{m^{2}} G_{1}^{\pi N}\left(Q^{2}\right)-\frac{i \sigma_{\mu \nu} q^{\nu}}{2 m} G_{2}^{\pi N}\left(Q^{2}\right)\right\} N(P),
\end{aligned}
$$

which can be related to the S-wave transverse $E_{0+}$ and longitudinal $L_{0+}$ multipoles:

$$
\begin{aligned}
& E_{0+}^{\pi N}=\frac{\sqrt{4 \pi \alpha_{\mathrm{em}}}}{8 \pi f_{\pi}} \sqrt{\frac{\left(2 m+m_{\pi}\right)^{2}+Q^{2}}{m^{3}\left(m+m_{\pi}\right)^{3}}}\left(Q^{2} G_{1}^{\pi N}-\frac{1}{2} m m_{\pi} G_{2}^{\pi N}\right), \\
& L_{0+}^{\pi N}=\frac{\sqrt{4 \pi \alpha_{\mathrm{em}}}}{8 \pi f_{\pi}} \frac{m\left|\omega_{\gamma}^{\mathrm{th}}\right|}{2} \sqrt{\frac{\left(2 m+m_{\pi}\right)^{2}+Q^{2}}{m^{3}\left(m+m_{\pi}\right)^{3}}}\left(G_{2}^{\pi N}+\frac{2 m_{\pi}}{m} G_{1}^{\pi N}\right) .
\end{aligned}
$$

The differential cross section at threshold is given by

$$
\left.\frac{d \sigma_{\gamma^{*}}}{d \Omega_{\pi}}\right|_{\mathrm{th}}=\frac{2\left|\vec{k}_{f}\right| W}{W^{2}-m^{2}}\left[\left(E_{0+}^{\pi N}\right)^{2}+\varepsilon \frac{Q^{2}}{\left(\omega_{\gamma}^{\mathrm{th}}\right)^{2}}\left(L_{0+}^{\pi N}\right)^{2}\right] .
$$

Here and below $m=939 \mathrm{MeV}$ is the nucleon mass, $W^{2}=\left(k+P^{\prime}\right)^{2}$ is the invariant energy, $\vec{k}_{f}$ and $\omega_{\gamma}^{\text {th }}$ are the pion three-momentum and the photon energy in the c.m. frame. The generalised form factors in (1.2) are real functions of the momentum transfer $Q^{2}$ at the threshold $W=m+m_{\pi}$. For generic $W$ the definition in (1.2) can be extended to specify two of the existing six invariant amplitudes, $G_{1,2}\left(Q^{2}\right) \rightarrow G_{1,2}\left(Q^{2}, W\right)$, which become complex functions.

The celebrated low-energy theorem (LET) $[3,4,5]$ relates the $S$-wave multipoles or, equivalently, the form factors $G_{1}, G_{2}$ at threshold, to the nucleon electromagnetic and axial form factors for vanishing pion mass $m_{\pi}=0$

$$
\begin{aligned}
& \frac{Q^{2}}{m^{2}} G_{1}^{\pi^{0} p}=\frac{g_{A}}{2} \frac{Q^{2}}{\left(Q^{2}+2 m^{2}\right)} G_{M}^{p}, \quad G_{2}^{\pi^{0} p}=\frac{2 g_{A} m^{2}}{\left(Q^{2}+2 m^{2}\right)} G_{E}^{p}, \\
& \frac{Q^{2}}{m^{2}} G_{1}^{\pi^{+} n}=\frac{g_{A}}{\sqrt{2}} \frac{Q^{2}}{\left(Q^{2}+2 m^{2}\right)} G_{M}^{n}+\frac{1}{\sqrt{2}} G_{A}, \quad G_{2}^{\pi^{+} n}=\frac{2 \sqrt{2} g_{A} m^{2}}{\left(Q^{2}+2 m^{2}\right)} G_{E}^{n} .
\end{aligned}
$$

Here the terms in $G_{M, E}$ are due to pion emission off the initial proton state, whereas for charged pion in addition there is a contribution corresponding to the chiral rotation of the electromagnetic current.

The subsequent discussion concentrated mainly on the corrections to (1.5) due to finite pion mass $[6,7]$. More recently, the threshold pion production for small $Q^{2}$ was reconsidered and the low-energy theorems re-derived in the framework of the chiral perturbation theory (CHPT), see [8] for a review. The new insight gained from CHPT calculations [9] is that the expansion at small 
$Q^{2}$ has to be done with care as the limits $m_{\pi} \rightarrow 0$ and $Q^{2} \rightarrow 0$ do not commute, in general. The LET predictions seem to be in good agreement with experimental data on pion photoproduction [10], However, it appears [11, 8] that the S-wave electroproduction cross section (1.4) for already $Q^{2} \sim 0.1 \mathrm{GeV}^{2}$ cannot be explained without taking into account chiral loops.

For larger momentum transfers the situation is much less studied as the power counting of CHPT cannot be applied. The traditional derivation of LET using PCAC and current algebra does not seem to be affected as long as the emitted pion is 'soft' with respect to the initial and final state nucleons simultaneously. The corresponding condition is, parametrically, $Q^{2} \ll \Lambda^{3} / m_{\pi}$ (see, e.g. [6]) where $\Lambda$ is some hadronic scale, and might be satisfied for $Q^{2} \sim 1 \mathrm{GeV}^{2}$ or even higher. We are not aware of any dedicated analysis of the threshold production in the $Q^{2} \sim 1 \mathrm{GeV}^{2}$ region, however.

It was suggested in Ref. [12] that in the opposite limit of very large momentum transfers the standard pQCD collinear factorisation approach $[13,14]$ becomes applicable and the helicityconserving $G_{1}^{\pi N}$ form factor can be calculated for $m_{\pi}=0$ in terms of chirally rotated nucleon distribution amplitudes. In practice one expects that the onset of this regime is postponed to very large momentum transfers because the factorisable contribution involves a small factor $\alpha_{s}^{2}(Q) / \pi^{2}$ and has to win over nonperturbative "soft" contributions that are suppressed by an extra power of $Q^{2}$ but do not involve small coefficients.

The purpose of this study is to suggest a realistic QCD-motivated model for the $Q^{2}$ dependence of the $G_{1,2}$ form factors alias S-wave multipoles at threshold in the region $Q^{2} \sim 1-10 \mathrm{GeV}^{2}$ that can be accessible in current and future experiments in Jefferson Laboratory and elsewhere (HERMES, MAMI).

\section{Light-Cone-Sum Rules}

In Ref. [15] we have developed a technique to calculate baryon form factors for moderately large $Q^{2}$ using light-cone sum rules (LCSR) $[16,17]$. This approach is attractive because in LCSRs "soft" contributions to the form factors are calculated in terms of the same nucleon distribution amplitudes (DAs) that enter the pQCD calculation and there is no double counting. Thus, the LCSRs provide one with the most direct relation of the hadron form factors and distribution amplitudes that is available at present, with no other nonperturbative parameters.

The same technique can be applied to pion electroproduction. In Refs. [1, 2] the $G_{1}$ and $G_{2}$ form factors were estimated in the LCSR approach for the range of momentum transfers $Q^{2} \sim 2-10 \mathrm{GeV}^{2}$. A new technical element in these calculations is taking into account the semidisconnected pion-nucleon contributions in the intermediate state. We demonstrate that, with this addition, the LET results in (1.5) are indeed reproduced at $Q^{2} \sim 1 \mathrm{GeV}^{2}$ to the required accuracy $\mathscr{O}\left(m_{\pi}\right)$, whereas the pQCD contribution considered in [12] formally corresponds to the leading (at large $Q^{2}$ ) part of the NNLO radiative correction $\sim \mathscr{O}\left(\alpha_{s}^{2}\right)$ to the sum rules. Hence our approach decribes both the high- $Q^{2}$ and the low- $Q^{2}$ limit correction and presents a QCD-motivated model at intermediate momenta that makes maximal use of quark-hadron duality and dispersion relations.

Accurate quantitative predictions are difficult for several reasons, one of them being that the nucleon distribution amplitudes are poorly known. In order to minimise the dependence of various parameters in this work one can try to use the LCSRs to predict certain form factor ratios and then 

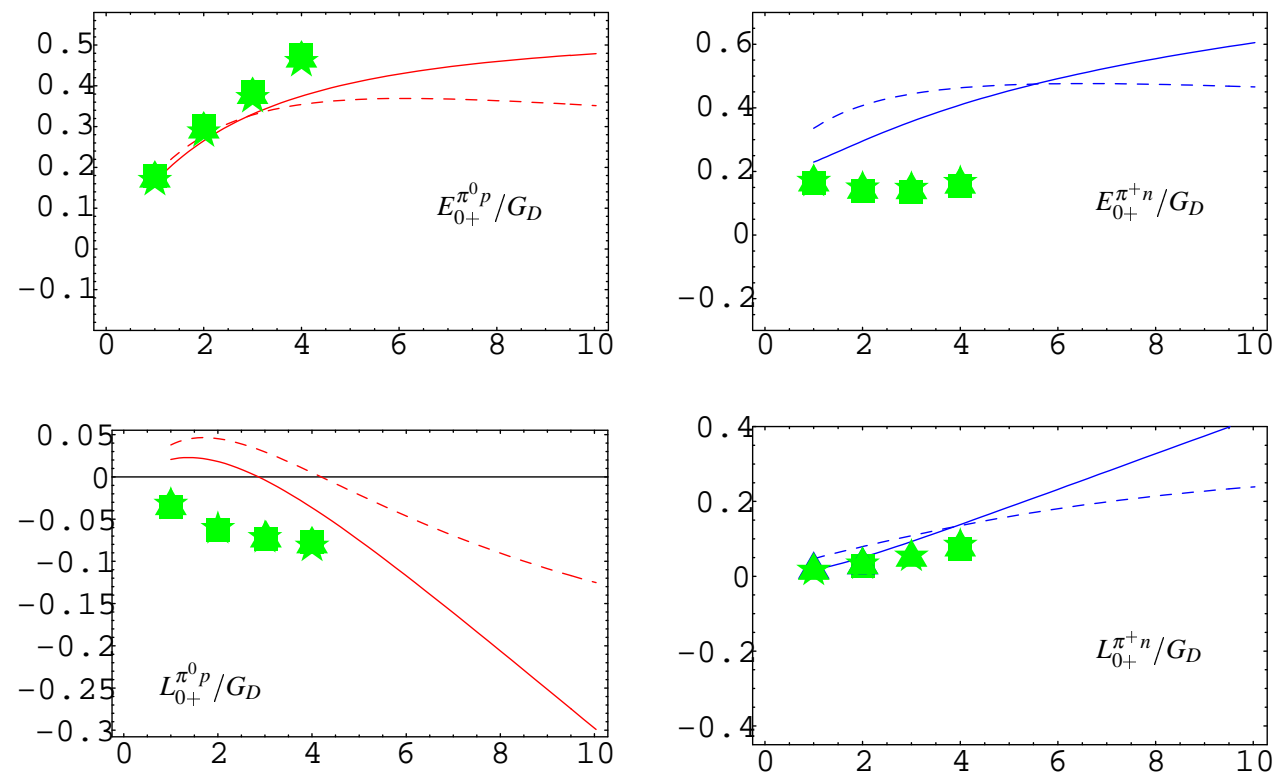

Figure 1: The LCSR-based model (solid curves) for the $Q^{2}$ dependence of the electric and longitudinal partial waves at threshold $E_{0+}$ and $L_{0+}$, in units of $\mathrm{GeV}^{-1}$, normalised to the dipole formula compared to MAID07 [22].

normalise to the electromagnetic nucleon form factors as measured in the experiment, see Refs. [2] for the details. In particular we use the parametrisation of the proton magnetic form factor from [18] and for the neutron magnetic form factor from [19]. For the proton electric form factor we use the fit $[20,18]$ to the combined JLab data in the $0.5<Q^{2}<5.6 \mathrm{GeV}^{2}$ range $\mu_{p} \frac{G_{E}^{p}}{G_{M}^{p}}=1-0.13\left(Q^{2}-0.04\right)$ and put the neutron electric form factor to zero, which is sufficient to our accuracy.

The resulting LCSR-based model is shown by the solid curves in Fig. 1, where the partial waves at threshold that are related to the generalised form factors through the Eq. (1.3) are plotted as a function of $Q^{2}$, normalised to the dipole formula $G_{D}\left(Q^{2}\right)=1 /\left(1+Q^{2} / \mu_{0}^{2}\right)^{2}$ where $\mu_{0}^{2}=0.71$ $\mathrm{GeV}^{2}$. To give a rough idea about possible uncertainties, the "pure" LCSR predictions (all form factors and other input taken from the sum rules) are shown by dashed curves for comparison. These models are used in the numerical analysis presented below. We expect that our present accuracy is about $50 \%$. It can be improved in future by the calculation of radiative corrections to the LCSRs and if sufficiently accurate lattice calculations of the moments of nucleon distribution amplitudes become available (cf. [21]).

\section{Moving Away From Threshold}

As a simple approximation, we suggest to calculate pion production near threshold in terms of the generalised form factors (1.2) and taking into account pion emission from the final state which dominates the P-wave contribution in the chiral limit (cf.[12]). In particular, we use the following expression:

$$
\left\langle N\left(P^{\prime}\right) \pi(k)\left|j_{\mu}^{e m}(0)\right| p(P)\right\rangle=
$$




$$
\begin{aligned}
= & -\frac{i}{f_{\pi}} \bar{N}\left(P^{\prime}\right) \gamma_{5}\left\{\left(\gamma_{\mu} q^{2}-q_{\mu} \not\right) \frac{1}{m^{2}} G_{1}^{\pi N}\left(Q^{2}\right)-\frac{i \sigma_{\mu \nu} q^{v}}{2 m} G_{2}^{\pi N}\left(Q^{2}\right)\right\} N(P) \\
& +\frac{i c_{\pi} g_{A}}{\left.2 f_{\pi}\left[\left(P^{\prime}+k\right)^{2}\right)-m^{2}\right]} \bar{N}\left(P^{\prime}\right) \not k \gamma_{5}\left(\not P^{\prime}+m\right)\left\{F_{1}^{p}\left(Q^{2}\right)\left(\gamma_{\mu}-\frac{q_{\mu} \not q}{q^{2}}\right)\right. \\
& \left.+\frac{i \sigma_{\mu \nu} q^{v}}{2 m} F_{2}^{p}\left(Q^{2}\right)\right\} N(P) .
\end{aligned}
$$

Here $F_{1}^{p}\left(Q^{2}\right)$ and $F_{2}^{p}\left(Q^{2}\right)$ are the Dirac and Pauli electromagnetic form factors of the proton, $c_{\pi^{0}}=$ 1 and $c_{\pi^{+}}=\sqrt{2}$ is the isospin factor, $g_{A}=1.267$ and $f_{\pi}=93 \mathrm{MeV}$.

The separation of the generalised form factor contribution and the final state emission in (3.1) can be justified in the chiral limit $m_{\pi} \rightarrow 0$ but involves ambiguities in contributions $\sim \mathscr{O}\left(m_{\pi}\right)$. We have chosen not to include the term $\sim \not k$ in the numerator of the proton propagator in the third line in (3.1) so that this contribution strictly vanishes at the threshold. In addition, we found it convenient to include the term $\sim q_{\mu} \not / q^{2}$ in the Lorentz structure that accompanies the $F_{1}$ form factor in order to make the amplitude formally gauge invariant. To avoid misunderstanding, note that our expression is not suitable for making a transition to the photoproduction limit $Q^{2}=0$ in which case, e.g. pion radiation from the initial state has to be taken in the same approximation to maintain gauge invariance.

The virtual photon cross section can be written as a sum of terms

$$
d \sigma_{\gamma^{*}}=\frac{\alpha_{\mathrm{em}}}{8 \pi} \frac{k_{f}}{W} \frac{d \Omega_{\pi}}{W^{2}-m^{2}}\left|\mathscr{M}_{\gamma^{*}}\right|^{2}
$$

with

$$
\begin{aligned}
\left|\mathscr{M}_{\gamma^{*}}\right|^{2}= & M_{T}+\varepsilon M_{L}+\sqrt{2 \varepsilon(1+\varepsilon)} M_{L T} \cos \left(\phi_{\pi}\right) \\
& +\varepsilon M_{T T} \cos \left(2 \phi_{\pi}\right)+\lambda \sqrt{2 \varepsilon(1-\varepsilon)} M_{L T}^{\prime} \sin \left(\phi_{\pi}\right) ;
\end{aligned}
$$

in the last term $\lambda$ is the beam helicity.

The complete expressions for the invariant functions are rather cumbersome but are simplified significantly in the chiral limit $m_{\pi} \rightarrow 0$ and assuming $k_{f}=\mathscr{O}\left(m_{\pi}\right)$. We obtain

$$
\begin{aligned}
f_{\pi}^{2} M_{T}= & \frac{4 \vec{k}_{i}^{2} Q^{2}}{m^{2}}\left|G_{1}^{\pi N}\right|^{2}+\frac{c_{\pi}^{2} g_{A}^{2} \vec{k}_{f}^{2}}{\left(W^{2}-m^{2}\right)^{2}} Q^{2} m^{2} G_{M}^{2} \\
& +\cos \theta \frac{c_{\pi} g_{A}\left|k_{i}\right|\left|k_{f}\right|}{W^{2}-m^{2}} 4 Q^{2} G_{M} \operatorname{Re} G_{1}^{\pi N}, \\
f_{\pi}^{2} M_{L}= & \vec{k}_{i}^{2}\left|G_{2}^{\pi N}\right|^{2}+\frac{4 c_{\pi}^{2} g_{A}^{2} \vec{k}_{f}^{2}}{\left(W^{2}-m^{2}\right)^{2}} m_{N}^{4} G_{E}^{2} \\
& -\cos \theta \frac{c_{\pi} g_{A}\left|k_{i}\right|\left|k_{f}\right|}{W^{2}-m^{2}} 4 m^{2} G_{E} \operatorname{Re} G_{2}^{\pi N}, \\
f_{\pi}^{2} M_{L T}= & -\sin \theta \frac{c_{\pi} g_{A}\left|k_{i}\right|\left|k_{f}\right|}{W^{2}-m^{2}} Q m\left[G_{M} \operatorname{Re} G_{2}^{\pi N}+4 G_{E} \operatorname{Re} G_{1}^{\pi N}\right], \\
f_{\pi}^{2} M_{T T}= & 0, \\
f_{\pi}^{2} M_{L T}^{\prime}= & -\sin \theta \frac{c_{\pi} g_{A}\left|k_{i}\right|\left|k_{f}\right|}{W^{2}-m^{2}} Q m\left[G_{M} \operatorname{I} m G_{2}^{\pi N}-4 G_{E} \operatorname{I} m G_{1}^{\pi N}\right],
\end{aligned}
$$


where $\vec{k}_{i}$ is the c.m.s. momentum in the initial state. Note that the single spin asymmetry contribution $\sim M_{L T}^{\prime}$ involves imaginary parts of the generalised form factors that arise because of the final state interaction. In our approximation $M_{T T}=0$ which is because we do not take into account the $\mathrm{D}$ - and higher partial waves. Consequently, the $\sim \cos (2 \phi)$ contribution to the cross section is absent.
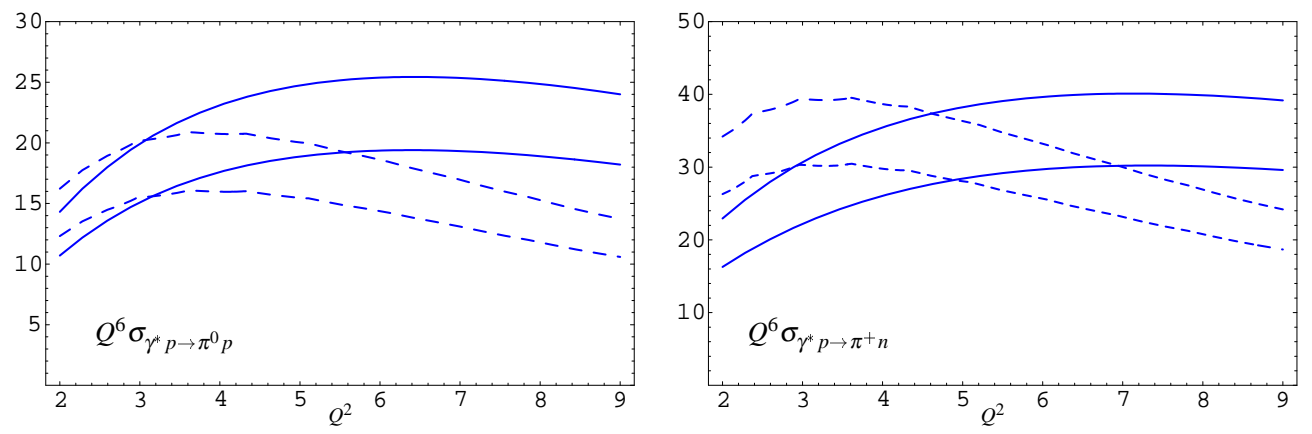

Figure 2: The integrated cross section $Q^{6} \sigma_{\gamma^{*} p \rightarrow \pi^{0} p}$ (left panel) and $Q^{6} \sigma_{\gamma^{*} p \rightarrow \pi^{+} n}$ (right panel) in units of $\mu b \times \mathrm{GeV}^{6}$ as a function of $Q^{2}$ for $W=1.11 \mathrm{GeV}$ (lower curves) and $W=1.15 \mathrm{GeV}$ (upper curves). The solid and the dashed curves correspond to the calculations using the two models for the partial waves at threshold $E_{0+}$ and $L_{0+}$ as shown in Figure. 1 (see text).

We find that the integrated cross sections scale like $\sigma_{\gamma^{*} p \rightarrow \pi N} \sim 1 / Q^{6}$, which is in agreement with the structure function measurements in the threshold region by E136 [23]. The S-wave contribution appears to be larger than P-wave up to $W \simeq 1.16 \mathrm{GeV}$. The ratio of $\pi^{0} p$ and $\pi^{+} n$ final states is approximately $1: 2$ and almost $Q^{2}$-independent, see Fig. 2

The comparison of our calculation for the structure function $F_{2}^{p}\left(W, Q^{2}\right)$ in the threshold region $W^{2}<1.4 \mathrm{GeV}^{2}$ to the SLAC E136 data [23] at the average value $Q^{2}=7.14 \mathrm{GeV}^{2}$ and $Q^{2}=$ $9.43 \mathrm{GeV}^{2}$ is shown in Fig. 3. The predictions are generally somewhat below these data $(\sim 50 \%)$, apart from the last data point at $W^{2}=1.4 \mathrm{GeV}^{2}$ which is significantly higher.
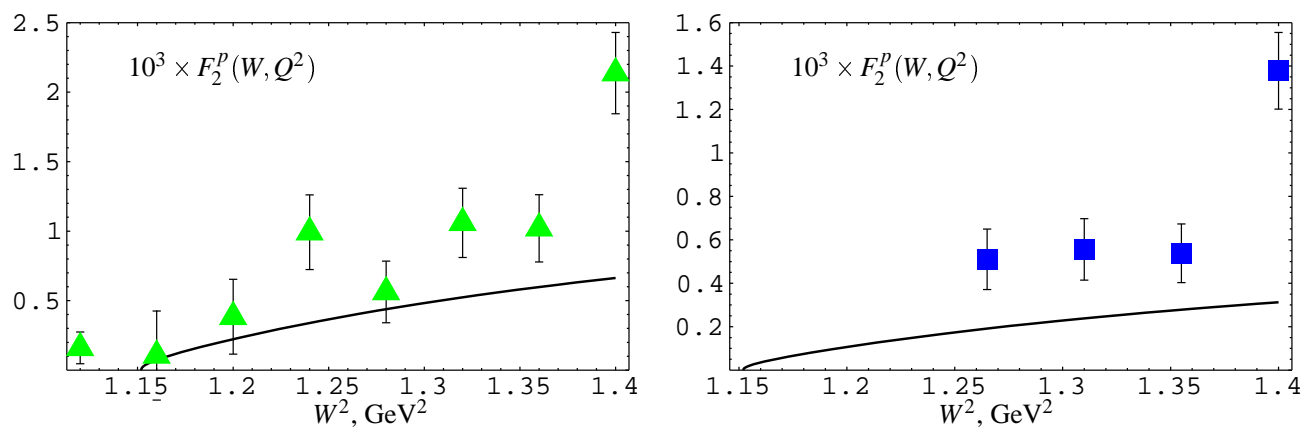

Figure 3: The structure function $F_{2}^{p}\left(W, Q^{2}\right)$ as a function of $W^{2}$ scaled by a factor $10^{3}$ compared to the SLAC E136 data [23] at the average value $Q^{2}=7.14 \mathrm{GeV}^{2}$ (left panel) and $Q^{2}=9.43 \mathrm{GeV}^{2}$ (right panel).

Note that in our approximation there is no D-wave contribution, and the final state interaction is not included. Both effects can increase the cross section so that we consider the agreement as satisfactory. We believe that the structure function at $W^{2}=1.4 \mathrm{GeV}^{2}$ already contains a considerable D-wave contribution and also one from the tail of the $\Delta$-resonance and thus cannot be compared with our model, at least in its present form. 
Finally, in Fig. 4 we present our predictions for $R=\sigma_{L} / \sigma_{T}$ for the $\pi^{+} n$ production.
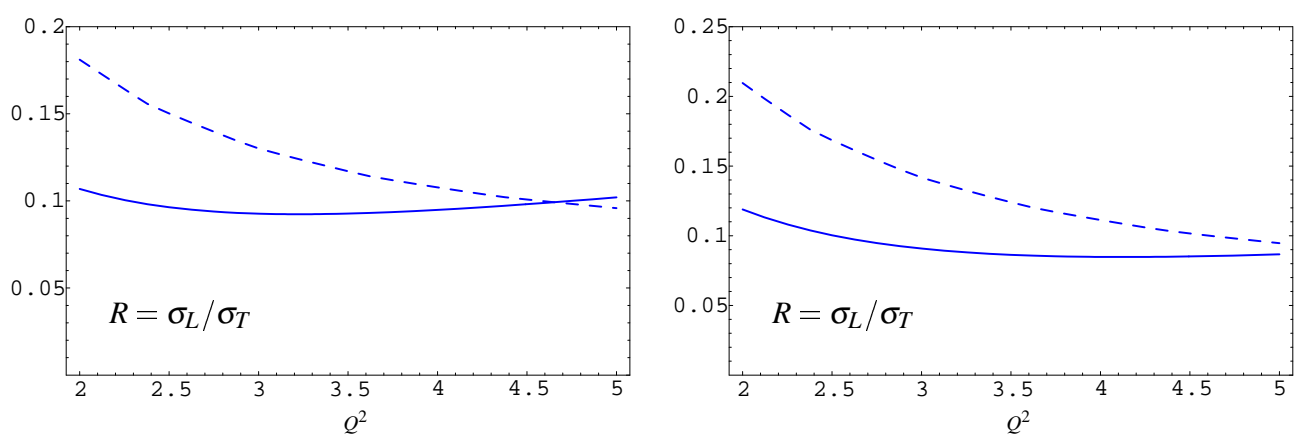

Figure 4: The $R=\sigma_{L} / \sigma_{T}$ ratio for $\gamma^{*} p \rightarrow \pi^{+} n$ production at $W=1.11 \mathrm{GeV}$ (left panel) and $W=1.15 \mathrm{GeV}$ (right panel). The solid and the dashed curves correspond to the calculations using the two models for the partial waves at threshold $E_{0+}$ and $L_{0+}$ as shown in Figure. 1 (see text).

To avoid misunderstanding we stress that the estimates of the cross sections presented here are not state-of-the-art and are only meant to provide one with the order-of-magnitude estimates of the threshold cross sections that are to our opinion most interesting. These estimates can be improved in many ways, for example taking into account the energy dependence of the generalised form factors generated by the FSI and adding a model for the D-wave contributions. The model can also be tuned to reproduce the existing lower $Q^{2}$ and/or larger $W$ experimental data.

\section{Acknowledgements}

We gratefully acknowledge discussions with A. Afanasev, V. Kubarovsky, A. Schäfer, P. Stoler and I. Strakovsky on various aspects of this project. V.B. thanks U. Meissner for bringing Ref. [9] to his attention and useful comments. The work of D.I. was partially supported by grants from RFBR-09-02-00263, NSh-1027.2008.2 and BMBF(06RY258). The work by A.P. was supported by the Studienstiftung des deutschen Volkes.

\section{References}

[1] V. M. Braun, D. Yu. Ivanov, A. Lenz and A. Peters, Phys. Rev. D 75, 014021 (2007).

[2] V. M. Braun, D. Yu. Ivanov and A. Peters, Phys. Rev. D 77 (2008) 034016.

[3] N. M. Kroll and M. A. Ruderman, Phys. Rev. 93, 233 (1954).

[4] Y. Nambu and D. Lurie, Phys. Rev. 125, 1429 (1962).

[5] Y. Nambu and E. Shrauner, Phys. Rev. 128, 862 (1962).

[6] A. I. Vainshtein and V. I. Zakharov, Nucl. Phys. B 36, 589 (1972).

[7] S. Scherer and J. H. Koch, Nucl. Phys. A 534, 461 (1991).

[8] V. Bernard, N. Kaiser and U. G. Meissner, Int. J. Mod. Phys. E 4, 193 (1995).

[9] V. Bernard, N. Kaiser and U. G. Meissner, Phys. Rev. Lett. 69, 1877 (1992).

[10] D. Drechsel and L. Tiator, J. Phys. G 18, 449 (1992). 
[11] V. Bernard, N. Kaiser, T. S. H. Lee and U. G. Meissner, Phys. Rev. Lett. 70, 387 (1993).

[12] P. V. Pobylitsa, M. V. Polyakov and M. Strikman, Phys. Rev. Lett. 87 (2001) 022001.

[13] A. V. Efremov and A. V. Radyushkin, Phys. Lett. B 94, 245 (1980).

[14] G. P. Lepage and S. J. Brodsky, Phys. Rev. D 22, 2157 (1980).

[15] V. M. Braun, A. Lenz, N. Mahnke and E. Stein, Phys. Rev. D 65, 074011 (2002); A. Lenz, M. Wittmann and E. Stein, Phys. Lett. B 581 (2004) 199; V. M. Braun, A. Lenz and M. Wittmann, Phys. Rev. D 73, 094019 (2006).

[16] I. I. Balitsky, V. M. Braun and A. V. Kolesnichenko, Nucl. Phys. B 312, 509 (1989).

[17] V. L. Chernyak and I. R. Zhitnitsky, Nucl. Phys. B 345, 137 (1990).

[18] E. J. Brash, A. Kozlov, S. Li and G. M. Huber, Phys. Rev. C 65, 051001 (2002).

[19] P. E. Bosted, Phys. Rev. C 51 (1995) 409; E. Tomasi-Gustafsson, F. Lacroix, C. Duterte and G. I. Gakh, Eur. Phys. J. A 24 (2005) 419.

[20] O. Gayou et al. [Jefferson Lab Hall A Collaboration], Phys. Rev. Lett. 88, 092301 (2002).

[21] V. M. Braun et al. [QCDSF Collaboration], Phys. Rev. D 79 (2009) 034504.

[22] D. Drechsel, S. S. Kamalov and L. Tiator, Eur. Phys. J. A 34, 69 (2007).

[23] P. E. Bosted et al., Phys. Rev. D 49, 3091 (1994). 